\title{
EXPERIÊNCIA BRASILEIRA INICIAL EM CIRURGIA DA OBESIDADE ROBÔ- ASSISTIDA
}

\author{
Brazilian experience in obesity surgery robot-assisted \\ Ricardo Zugaib ABDALLA, Rodrigo Biscuola GARCIA, Claudio Renato Penteado de LUCA, \\ Rafael Izar Domingues da COSTA, Claudia de Oliveira COZER
}

Trabalho realizado no Hospital Sírio Libanês, São Paulo,SP, Brasil.

DESCRITORES - Obesidade/cirurgia. Robótica. Procedimentos cirúrgicos minimamente invasivos.
RESUMO - Racional - As técnicas minimamente invasivas associaram-se à cirurgia bariátrica propiciando redução na agressão aos órgãos e sistemas. A robótica é uma nova possibilidade dentro do conceito de mínima invasão. Objetivos - Avaliar a experiência inicial da robótica em cirurgia da obesidade. Métodos - Foram operados 27 pacientes no mesmo hospital, pela mesma equipe, com o mesmo sistema cirúrgico que foi Da Vinci S. Resultados - Foram realizadas seis colocações de banda gástrica, cinco gastrectomias verticais e 16 septações gástricas com by-pass em Y-de-Roux. Ocorreu uma complicação importante e nenhum óbito. Conclusões - A via robótica mostrou-se segura durante experiência inicial, propiciando maior facilidade durante o procedimento em pacientes obesos.

\section{Correspondência:}

Ricardo Zugaib Abdalla,

e-mail: ricardo.abdalla@hsl.org.br

Fonte de financiamento: não há

Conflito de interesses: não há

Recebido para publicação:

Aceito para publicação:

HEADINGS - Obesity/surgery. Robotics. Surgical procedures, minimally invasive.
ABSTRACT - Background - Minimally invasive techniques were associated with bariatric surgery, leading to a decrease in aggression to the organs and systems. Robotic surgery is a new possibility within the concept of minimal invasion. Aim - To evaluate the initial experience of robotic surgery for obesity. Methods - Twenty seven patients were operated in the same hospital, by the same team with the same surgical system, Da Vinci S. Results - Were performed six gastric band placement, five sleeve gastrectomies and 16 gastric bypasses. There was one major complication and no death in this series. Conclusions - The robotic surgery for obesity surgery was safe during the initial experience.

\section{INTRODUÇÃO}

$\mathrm{O}$ ideal da cirurgia bariátrica é trazer ao paciente obeso mórbido significante índice de perda de peso a curto e longo prazo, com o mínimo possível de complicações operatórias.

As técnicas minimamente invasivas associaram-se à cirurgia bariátrica e propiciaram grande redução na agressão aos órgãos e sistemas, com diminuição da morbidade e do tempo de recuperação. Existe significante melhora das complicações pulmonares e de ferida operatória, além de diminuição no tempo de internação hospitalar? .

A primeira aplicação da robótica em cirurgia bariátrica é atribuída à Cadiere, que em 1999 instalou uma banda gástrica robô - assistida². Em 2003 Jacobsen por sua vez publicou estudo de 107 casos de by-pass gástrico operados por 11 cirurgiões diferentes. Nesta amostra não houve nenhuma deiscência de anastomose gastrojejunal, sendo que as vantagens apontadas deste procedimento foram a visão em $3-D$, a possibilidade de confecção de um pouch menor e o menor uso de grampeadores devido à facilidade da realização de anastomoses com o robô. Em compensação, o tempo cirúgico foi maior com a robótica, em relação à cirurgia laparoscópica e laparotômica ${ }^{3}$. 
Mais trabalhos surgem para avaliar a curva de aprendizado em cirurgia bariátrica robótica. Para tal, é usada uma unidade na qual o tempo cirúrgico é dividido pelo índice de massa corpórea do paciente, chegando em um resultado expresso em minutos/IMC. Mohr, em 2005, comparou o tempo cirúrgico de suas primeiras gastroplastias robóticas com uma amostra retrospectiva das mesmas operações laparoscópicas e chegou à conclusão que a cirurgia robótica era mais rápida (3,8 min/unidade IMC versus $5,0 \mathrm{com} p=0,009)^{5}$.

Em 2005 Dominick Artuso, em Nova Iorque, fez uma análise de 41 gastroplastias robóticas realizadas entre 2001 e 2002, comparando com a laparoscopia. O tempo cirúrgico mostrou-se maior com o robô, sem diferença estatística em relação ao número de deiscências de anastomose gastrojejunal ${ }^{1,6}$.

Os americanos conseguiram analisar grande número de pacientes, graças à grande disponibilidade dos sistemas cirúrgicos robóticos naquele país. Em 2008, o Huntington Memorial Hospital em Pasadena, publicou uma análise de 100 casos de by-pass laparoscópicos robô-assistidos, chegando à conclusão que a realização do procedimento era segura em um hospital comunitário, tendo resultados não inferiores à gastroplastia laparoscópica nas mãos de cirurgiões experientes ${ }^{4}$. Além de séries maiores, existem exemplos de pacientes com obesidade extrema tratados com o uso da robótica, tal como relato de neoplasia ginecológica operada através de cirurgia robô-assistida em uma paciente com IMC de 98. Este exemplo ilustra bem a sensação de facilidade que o sistema robótico traz na operação dos pacientes obesos em relação à laparoscopia tradicional ${ }^{8}$.

Nos países europeus o volume de cirurgias bariátricas realizadas por via robô-assistida ainda é bem menor do que nos EUA. Os estudos são na maioria relatos de casos e pequenas séries mostrando a experiência inicial de alguns centros, com poucos casos . Exemplo disso é a série do grupo de Atenas, na Grécia, com uma análise de nove casos operados entre dezembro de 2008 e maio de 2009.

Outras técnicas foram estudadas, tal como a banda gástrica por robótica. Horgan em 2004 chegou à conclusão que a via laparoscópica convencional era mais rápida para colocação da banda, porém a via robótica teve menor morbidade com o mesmo tempo médio de internação hospitalar. Segundo o autor, o robô facilitava a confecção de suturas difíceis, principalmente nos pacientes super-obesos ${ }^{6}$.

O objetivo deste trabalho foi descrever a experiência pioneira de equipe já atuante em cirurgia da obesidade, com a nova via de acesso robô- assistida.

MÉTODOS

Foi realizado, através do sistema Da Vinci $S$, um total de 27 operações bariátricas pelo mesmo cirurgião entre setembro de 2008 e agosto de 2011 no Hospital Sírio-Libanês em São Paulo, SP, Brasil. Dentre as operações executaram-se seis colocações de banda gástrica, cinco gastrectomias verticais e 16 septações gástricas com by-pass em Y-de-Roux. Foram avaliadas complicações técnicas do método, infecção da ferida operatória, hérnia incisional, re-operações e mortalidade

\section{RESULTADOS}

Ao todo foram operados 10 homens e 17 mulheres. Três gastrectomias verticais foram realizadas em pacientes do sexo masculino e duas em pacientes do sexo feminino. Foram instaladas bandas gástricas em dois homens e quatro mulheres. Os by-pass foram realizados em cinco homens e 11 mulheres.

Em quatro pacientes que realizaram by-pass gástrico e uma que realizou gastrectomia vertical, ocorreram hematomas nos pontos de entrada dos portais. Nenhum desses pacientes evoluiu com infecção da ferida operatória, e nenhum até o momento apresentou hérnia incisional.

Ocorreu uma complicação importante do procedimento, devido à lesão inadvertida de intestino delgado durante o grampeamento da enteroenteroanastomose. A lesão foi identificada no segundo dia do pós-operatório e tratada com laparotomia e enterectomia.

Não houve outra reoperação na série descrita como também complicações tardias até o momento deste publicação. Nenhum paciente morreu.

\section{DISCUSSÃO}

A cirurgia bariátrica robô-assistida oferece vantagens principalmente ao cirurgião. $O$ fato de se operar com ergonomia adequada e de forma confortável traz ao profissional mais tranquilidade na realização de procedimentos mais desgastantes. Entre esses procedimentos pode ser incluída a cirurgia da obesidade, que eventualmente pode ter duração aumentada e proporcionar ao cirurgião grande esforço físico.

O grupo em questão é pioneiro na realização de cirurgia da obesidade robô - assistida no Brasil, tendo realizado os primeiros procedimentos no país e até o momento tem a maior experiência brasileira no assunto. $O$ primeiro procedimento realizado foi um bypass gástrico em julho de 2008.

Nesta casuística é importante lembrar que a complicação ocorrida foi relacionada às linhas de grampeamento realizadas, e ocorreu no primeiro procedimento. O grampeamento na cirurgia robótica é realizado pelo auxiliar, através de trocarte de laparoscopia comum, sem utilização de material próprio do robô. Desta forma não se pode considerar 
a deiscência da enteroenteroanastomose uma complicação do método robótico em si. Por outro lado, mostra que o assistente na cirurgia robótica tem papel importante, devendo ser devidamente treinado para procedimentos avançados de videocirurgia, tal como grampeamento da neocâmara no by-pass gástrico.

O que a literatura mostra é que a cirurgia bariátrica robótica tem espaço, principalmente em equipes bem treinadas e hospitais preparados para tal. A superioridade da cirurgia abdominal robô-assistida ainda não foi claramente demonstrada, porém a literatura sobre o assunto vem crescendo, inclusive com o surgimento da telecirurgia e cirurgia à distância ${ }^{1,4}$.

A impressão inicial, até no momento empírica, é que o robô facilita procedimentos a princípio mais complicados, tais como operações em pacientes superobesos. Além disso, possibilita a gastroenteroanatomose manual (com uso do braço robótico) calibrada, oferecendo mais precisão no procedimento. Vencida a curva de aprendizado e com toda a equipe treinada e certificada em cirurgia robótica o procedimento tornase tranquilo e padronizado.

\section{CONCLUSÃO}

A cirurgia robô-assistida facilita procedimentos em pacientes obesos. Como a experiência inicial, pode-se concluir que o método é seguro e deve ser mais estudado através de trabalhos comparativos com a videolaparoscopia, que hoje é realizada com mais frequência em grandes centros.

\section{REFERÊNCIAS}

1. Artuso D, Wayne M, Grossi R. Use of Robotics During Laparoscopic Gastric Bypass for Morbid Obesity. JSLS .2005; 9:266-268

2. Cadiere GB, Himpens J, Vertruyen M, Favretti F. The world's first obesity surgery performed by a surgeon at a distance. Obes surg .1999; 9: 206-209

3. Jacobsen G, Berger R, Horgan $S$. The role of robotic surgery in morbid obesity. Journal of laparoendoscopic \& advanced surgical techniques. 2003; 13(4): 279-283.

4. Joseph Y, Deng MD, Da Vid J, Lourié MD, 100 Robotic-Assisted Laparoscopic Gastric Bypasses at a Community Hospital. The American Surgeon. 2008 ; 74 (10): $1022-25$

5. Mohr CJ, Nadzam GS, Alami RS, Sanchez BR, Curet MJ. Totally robotic laparoscopic Roux-en-Y Gastric bypass: results from 75 patients. Obesity Surgery. 2006;16(6):690-6.

6. Moser F, Horgan S. Robotically assisted bariatric surgery. American Journal of Surgery. 2004. 188 (4a suppl): 38S-44S.

7. Nguyen NT, Goldman C, Rosenquist CJ, Arango A, Cole CJ, Lee SJ, Wolfe BM. Laparoscopic versus open gastric bypass: a randomized study of outcomes, quality of life, and costs. Ann Surg. 2001; 234(3):279-289.

8. Stone P, Burnett A, Burton B, Roman J. Overcoming extreme obesity with robotic surgery. Int J Med Robotics Comput Assist Surg .2010. Published online in Wiley InterScience (www.interscience.wiley. com). DOI: 10.1002/rcs.341 